\section{Manipulative begging by parasitic cuckoo nestlings and paradoxical host behaviour}

In his News \& Comment $^{1}$ on a study by Davies et al. about begging in cuckoos², Lotem raises issues that deserve further attention. Davies et al. identified the begging call of European cuckoo (Cuculus canorus) nestlings, resembling not a nestling but an entire host brood as the necessary and sufficient stimulus to make their reed warbler (Acrocephalus scirpaceus) hosts provision enough food to fulfil the cuckoo's requirements (roughly those of a whole brood of hosts), despite being the sole occupant of the nest (and one strikingly different from host young).

It is puzzling that hosts even tolerate the parasite, let alone favour it. According to the Manipulative Interference Model ${ }^{3,4}$ (MIM), those parasites handicapped by their odd appearance or numerical inferiority could compensate for their handicap, eliciting preferential care by hosts by means of manipulative signals. Traditionally, the MIM focused on signals exaggerating traits indicative of a high nestling quality, in particular nestlings with higher fitness returns per unit care, such as larger nestlings or those in greater food need (for which reason it is also known, less properly, as the super-normal stimulus hypothesis $\left.{ }^{1-5}\right)$. Like Davies et al. ${ }^{2}$, Lotem ${ }^{1}$ argues that this recent cuckoo study provides a different answer from the traditional one to the problem of host preference. Namely, cuckoos pretend to be several normal nestlings instead of a single highquality one. However, the new discovery is not inconsistent with the MIM model. In fact, although the specific trick used by the cuckoo is different from the traditional chick-quality signals, the essence of the behaviour is exactly as predicted by the MIM. In strictly functional terms, it makes no difference whether cuckoos exploit adaptive parental rules for preferential feeding according to offspring number, size or need. The MIM neither limits the range of traits that can be exaggerated, nor even requires super-normal signals (cf. Ref. 5), nor exploitation of adaptive rules to invoke costs that maintain the equilibrial absence of host rejection. Any other mechanism causing a compensating preferential response (say, hidden sensory preference ${ }^{6}$ ) that was resistant to evolutionary modification (because of, for example, perceptual constraints) would work equally. I agree with Lotem ${ }^{1}$ in that unambiguous evidence for the MIM is still lacking, but in the sense of whether manipulative begging is a coevolved anti-rejection response. This, and at least two more studies ${ }^{4,7}$, show that exaggerated begging by parasites handicapped by their small number ${ }^{2}$ and size $^{4}$, or dissimilar appearance ${ }^{7}$, helps them to prevent suboptimal feeding by hosts, a likely chick-rejection response actually reported in chick-discriminating hosts ${ }^{4,7}$.

It is no less intriguing why host nestlings refrain from begging at a similar, cuckoo-like intensity to get extra food. As Lotem ${ }^{1}$ remarks, we should expect nestlings to escalate begging intensity as an expression of genetic parent-offspring conflict ${ }^{8}$ but, I would argue, not necessarily above the actual level. There is no way to ascertain to what extent the currently observed begging level is, or can be further exaggerated, up to the maximum tolerable for the system to remain stable ${ }^{8}$. Aside from the most immediate explanation (non-zero genetic relatedness, $r$, among host young ${ }^{8}$ ), Lotem ${ }^{1}$ suggests that exaggerated begging benefits a cuckoo that is reared alone more than a host nestling sharing the brood with several nestmates because the latter will share any extra food provisioned but not the extra begging cost. It is wise to explore additional factors causing asymmetries in direct costs and benefits, so that we can rule them out before reasonably concluding any effect of $r$. However, as for $r$, the effect of food share is difficult to identify conclusively because cuckoo and warbler nestlings may differ in other relevant ways (e.g. size, growth or predation risk) likely to affect the begging benefit:cost ratio.

A better test may be provided by other cuckoohost species whose nestlings are similar in most respects, such as the great spotted cuckoo (Clamator glandarius) and its magpie (Pica pica) host. Great spotted cuckoo nestlings do not evict nest contents after hatching and thus can be reared alongside the similar-sized host young 9 . Here, neither direct (energetic and predation) costs nor food supply (both with regard to cuckoo/host relative size and to the presence of nestmates) can explain why magpies beg at low rates that honestly covary with need while cuckoos in similar conditions beg much more, irrespective of need ${ }^{4}$. A cuckoo and a mutant magpie endowed with a similar begging behaviour would do it equally well. This suggests a major role for $r$ as a key factor determining begging intensity and honesty.

\section{Reply from A. Lotem}

I welcome Redondo's clarification that Davies et al.'s new finding1 can be viewed as a specific case of his Manipulative Interference Model$^{2}$ (MIM). My suggestion that Davies et al.'s study provides a 'different answer' related to the precise way in which cuckoo chicks manipulate their hosts, not in questioning whether they do so.

Regarding the title question of my article 'Why should true offspring not do the same?': there is no doubt that because of the lack of genetic relatedness parasitic nestlings are expected to beg more ${ }^{3-5}$. However, because we cannot feasibly predict how much more, we cannot determine if this is the only reason; perhaps there are additional ones. Being satisfied with the immediate and obvious explanation, as suggested by Redondo, might not be the most productive methodology, because it weakens the motivation to consider alternatives or to look for additional factors.

Redondo is correct in predicting that my idea will not work in systems where the parasite is raised alongside the hosts' own young and cannot monopolize the benefit of its begging efforts. But perhaps this is the reason that mimicking a brood is performed by the single chicks of the common cuckoo ${ }^{1}$, rather than by parasites that compete with the host young2,6 If the suggested asymmetry can lead to

\section{Acknowledgements}

I am grateful to Fernando Alvarez, Juan Amat, Arnon Lotem, Miguel Rodriguez-Gironés and Jesus Zuñiga for many helpful criticisms, and to Nick Davies and David Noble for trusting my intuitions too.

\section{Tomas Redondo}

Estacion Biologica de Doñana,

CSIC, Apdo. 1056,

41080 Sevilla, Spain

(redondo@ebd.csic.es)

\section{References}

1 Lotem, A. (1998) Trends Ecol. Evol. 13, 342-343

2 Davies, N.B. et al. (1998) Proc. R. Soc. London Ser. B 256, 673-678

3 Dawkins, R. and Krebs, J.R. (1979) Proc. R. Soc. London Ser. B 205, 489-511

4 Redondo, T. (1993) Etologia 3, 235-297

5 Lichtenstein, G. and Sealy, S.G. (1998) Proc. R. Soc. London Ser. B 265, 249-254

6 Arak, A. and Enquist, M. (1993) Philos. Trans. R. Soc. London Ser. B 340, 207-213

7 Nicolai, J. (1969) J. Ornithol. 110, 421-447

8 Mock, D.W. and Parker, G.A. (1997) The Evolution of Sibling Rivalry, Oxford University Press

9 Soler, M. and Soler, J.J. (1991) Condor 93, 49-54

different manipulative strategies among brood parasites (where ' $r$ ' is always zero), a similar

effect on begging differences between a single cuckoo and a hostyoung is likely, irrespective of the difference in ' $r$ ' and its additional effect. More important, although the idea was inspired by the case of the cuckoo, such an asymmetry in securing a benefit share of collective begging might be found in many normal broods, where cuckoos and differences in ' $r$ ' are irrelevant.

\section{Arnon Lotem}

Dept of Zoology,

Faculty of Life Sciences,

Tel-Aviv University,

Tel-Aviv 69978, Israel

(lotem@post.tau.ac.il)

\section{References}

1 Davies, N.B. et al. (1998) Proc. R. Soc. London Ser. B 265, 673-678

2 Redondo, T. (1993) Etologia 3, 235-297

3 Johnstone, R.A. and Grafen, A. (1993) Anim. Behav. 46, 759-764

4 Briskie, J.V., Naugler, C.T. and Leech, S.M. (1994) Proc. R. Soc. London Ser. B 258, 73-78

5 Lotem, A. (1998) Trends Ecol. Evol. 13, 342-343

6 Lichtenstein, G. and Sealy, S.G. (1998) Proc. R. Soc. London Ser. B 265, 249-254 